\title{
Effect of combination of parenteral edaravone and nimodipine on ischemic cerebral injury following cerebral hemorrhage
}

\author{
Qiuju Li ${ }^{1}$, Zhiling $\mathrm{Ji}^{2}$, Kun $\mathbf{L i}^{3}$ \\ ${ }^{1}$ Department of Rehabilitation, Jining No. 1 People's Hospital, Jining City, ${ }^{2}$ Department of Neurology, Weifang Yidu Central \\ Hospital, Weifang City, ${ }^{3}$ Department of Emergency, Jining No. 1 People's Hospital, Jining City, Shandong Province, China
}

*For correspondence: Email: kt0749@163.com

Sent for review: 3 February 2018

Revised accepted: 27 April 2018

\begin{abstract}
Purpose: To study the clinical effects of parenteral edaravone when combined with nimodipine for the treatment of ischemic cerebral injury following cerebral hemorrhage, and its influence on $D$-dimer, brain natriuretic peptide (BNP) and transcription factor $k B(N F-k B)$ levels.

Methods: A total of 102 cases of ischemic cerebral injury after cerebral hemorrhage seen in 215 Hospital of Nuclear Industry in Shanxi, China from January, 2016 to January, 2017 were included in this study. They were randomly divided into two groups, viz, fifty-two cases in control group and fifty cases in observation group. Control group received nimodipine, while the observation group was given edaravone. Before and after treatment, the two groups' NIHSS scores were compared. Adverse reactions were recorded, and clinical effectiveness was evaluated. Enzyme-linked immunosorbent assay (ELISA) was used to determin thee levels of serum D-dimmer, BNP and NF-kB in the two groups. Results: Before treatment, the two groups' NIHSS scores did not show significant differences $(p>$ 0.05). However, after treatment, the observation group's NIHSS score was significantly lower than that of the control group $(p<0.05)$. Total effectiveness in the observation and control groups were 98.0 and $80.8 \%$, respectively. Clinical effectiveness in patients in the observation group was significantly better than that of the control group $(p<0.05)$. Before treatment, $D$-dimmer, BNP and NF-kB levels of patients in the two groups showed no significant difference $(p>0.05)$. However, compared with the control group, D-dimmer, BNP and NF-kB levels in the observation group post-treatment decreased significantly $(p<0.05)$.

Conclusion: These results suggest that the combination of parenteral edaravone and nimodipine has relatively high efficiency and safety, mitigates cerebral ischemia and cerebral edema, and controls inflammatory reactions. Thus, its potentials in this regard requires further investigation.
\end{abstract}

Keywords: Edaravone, Nimodipine, Cerebral ischemia, Ischemic cerebral injury

This is an Open Access article that uses a funding model which does not charge readers or their institutions for access and distributed under the terms of the Creative Commons Attribution License (http://creativecommons.org/licenses/by/4.0) and the Budapest Open Access Initiative (http://www.budapestopenaccessinitiative.org/read), which permit unrestricted use, distribution, and reproduction in any medium, provided the original work is properly credited.

Tropical Journal of Pharmaceutical Research is indexed by Science Citation Index (SciSearch), Scopus, International Pharmaceutical Abstract, Chemical Abstracts, Embase, Index Copernicus, EBSCO, African Index Medicus, JournalSeek, Journal Citation Reports/Science Edition, Directory of Open Access Journals (DOAJ), African Journal Online, Bioline International, Open-J-Gate and Pharmacy Abstracts

\section{INTRODUCTION}

Due to increasing aging populations worldwide, cerebral stroke has become the second most important cause of death, and a major factor for disability. Primary cerebral hemorrhage is one of the most deadly strokes. Many survivors have various levels of disability. In China, cerebral hemorrhage accounts for 10 to $30 \%$ of all 
cerebral strokes, and is increasing year by year [1].

Studies have shown that the incidence of ischemic cerebral injury after cerebral hemorrhage is very high, accounting for 13 to 39 $\%$ in cerebral hemorrhage patients [2]. The mechanism involved in ischemic cerebral injury is still unclear. It may be related to rapid depressurization and microvascular lesions before incidence, and increased intracranial pressure. It has been reported that the incidence of adverse prognosis of ischemic cerebral injury after cerebral hemorrhage within one year increased 6 times [3]. Early prevention and treatment of ischemic cerebral injury after cerebral hemorrhage may exert a certain positive effect for improving prognosis of patients.

Nimodipine which is used to improve cerebral blood circulation and clinical symptoms of cerebral hemorrhage patients can achieve relatively ideal clinical effects [4]. Edaravone is a synthetic antioxidant agent that neutralizes free radicals; it relieves reperfusion injury associated with cerebral ischemia, inhibits oxidation of cerebral cells and improves cerebral ischemic symptoms [5].

The aim of the research was to study the effect of combining edaravone injection with nimodipine on the treatment of patients with ischemic cerebral injury after cerebral hemorrhage, and the effect of this combination on D-dimmer, BNP and NF levels. This was with a view to evaluating its safety and efficiency so as to provide basis for clinical treatment of the disease.

\section{EXPERIMENTAL}

\section{General characteristics of subjects}

Patients with ischemic cerebral injury after cerebral hemorrhage in the Department of Neurosurgery, 215 Hospital of Nuclear Industry in Shanxi from January, 2016 to January, 2017 were recruited as study subjects. They were randomly divided into two groups. All included patients met the diagnosis criteria of ischemic cerebral injury after cerebral hemorrhage as formulated by The Fourth National Conference on the Diagnosis of Cerebrovascular Disease Academic Standards [6]. The included patients, who had relatively long history of hypertension and atherosclerosis, patients who had sudden attacks such as headache, vomiting and disturbances in consciousness during physical activity or emotional excitement; patients who had neurological signs; and patients diagnosed with ischemic cerebral injury after cerebral hemorrhage by CT scan and MRI after hospital admission.

Vital signs were stable for patients in this group, and the compliance was good. Consciousness was clear, and the patients had no contraindications for edaravone and nimodipine. The following patients were excluded: patients who had excessive bleeding, multiple hematoma and hemorrhage of brain stem and cerebellum; patients who had ischemic cerebral injury caused by non-cerebral hemorrhage; and patients with severe liver and kidney dysfunction, psychological diseases or other organ dysfunctions.

This research was approved by the Ethical Committee of Jining NO.1 People's Hospital according to the declaration of Helsinki promulgated in 1964 as amended in 1996 [6], (approval number $=2017090302$ ). All patients signed informed consent form and were willing to receive relevant treatment and examination. A total of 102 patients met the inclusive criteria. There were 27 males and 23 females in observation group with mean age of $75.21 \pm 9.28$ years. There were 27 males and 25 females in the control group, with mean age of $76.48 \pm 3.58$ years.

\section{Treatment program}

Patients in the observation and the control group were given antibiotics and intracranial pressurelowering drugs. The observation group received $30 \mathrm{mg}$ edaravone (Sinopharm Group Guorui Pharmaceutical CO. Ltd; SFDA approval number $\mathrm{H} 20080056)$ by venous injection twice daily, and 120 mg nimodipine (Bayer Healthcare Co. Ltd.; SFDA approval number $\mathrm{H} 20003010)$ by oral administration three to four times. The control group was given $30 \mathrm{mg}$ edaravone by venous injection twice daily. The dosage of medication was adjusted according to conditions of patients in the two groups. Two weeks were one course. The total treatment time covered three courses.

\section{Observation indices}

The America National Institute of Health Stroke Scale (NIHSS) was used to evaluate the degree of nervous functional defects of patients in the two groups before and after treatment. The lower the score, the more normal the neurological function of patients. Before and after treatment, the levels of D-dimmer, BNP and NF-kB in two groups were determined by ELISA (Shanghai Enzyme-linked Biotech CO. Ltd). All adverse reactions of patients in the two groups were recorded during the medication process. 


\section{Criteria for evaluation of effects}

Obvious effects were: decrease in the nervous functional defects score of patients by over $90 \%$ and incidence of adverse reactions below $5 \%$. Validity covered nervous functional defects scores from 70 to $90 \%$, and incidence of adverse reactions below $10 \%$. Invalidity was confirmed by absence of decrease in nervous functional defects score, i.e., an increase in this score, and if patient's conditions showed no recovery. They were calculated as in Eqs 1,2 and 3.

$A(\%)=B+C$

Where $A$ is the total effective rate; $B$ is obvious effects; $C$ is validity

$B(\%)=(a / c) 100$

where $a$ is cases who decreased in the nervous functional defects score of patients by over $90 \%$ and incidence of adverse reactions below $5 \%$; c is the total cases

$C(\%)=(b / c) 100$

where $b$ is cases who covered nervous functional defects scores from 70 to $90 \%$; c is the total cases.

\section{Statistical analysis}

Data was analyzed by SPSS 22.0 software. Data are presented as mean \pm SD. Data before and after treatment were compared through paired ttest by SPSS 22.0 software (IBM Software, USA). $p<0.05$ showed that had statistical significance.

\section{RESULTS}

\section{General conditions of patients}

The data on age, sex and co-morbidities for the two groups showed no significant differences ( $p$ $>0.05$, Table 1).

\section{Nervous functional defect scores of patients}

Before treatment, nervous functional defects score for the two groups showed no significant differences $(p>0.05)$. However, compared with scores before treatment, two groups' NIHSS scores decreased after the three-course treatments. Compared with the score before treatment, that in the observation group was significantly better. At the same time, the score in the observation group also differed significantly from that in the control group $(p<0.05$, Table 2$)$.

Table 2: Nervous functional defects scores of patients before and after treatment (mean \pm SD)

\begin{tabular}{lccc}
\hline Group & Number & $\begin{array}{c}\text { Before } \\
\text { treatment }\end{array}$ & $\begin{array}{c}\text { After } \\
\text { treatment }\end{array}$ \\
\hline Observation & 50 & $26.02 \pm 1.48$ & $11.54 \pm 2.01$ \\
Control & 52 & $26.72 \pm 1.68$ & $18.06 \pm 2.28$ \\
$\mathrm{~T}$ & - & 0.571 & 10.161 \\
$P$ & - & 0.633 & 0.012 \\
\hline
\end{tabular}

Incidence of adverse reactions after treatment

There was one allergic reaction in the treatment group, manifesting in nausea and vomiting. The incidence of the adverse reactions was $2 \%$. In the control group, there was one allergic reaction and one local skin swelling; the incidence of the adverse reactions was $3.8 \%$. The incidence of adverse reactions in the control group was insignificantly higher than that in the observation group ( $p>0.05$; Table 3$)$.

Table 3: Incidence of adverse reactions after treatment of patients

\begin{tabular}{lcccc}
\hline \multirow{2}{*}{ Group } & Number & \multicolumn{2}{c}{ Adverse reactions } & Incidence \\
\cline { 3 - 4 } & & $\begin{array}{c}\text { Allergic } \\
\text { reactions }\end{array}$ & $\begin{array}{c}\text { Local } \\
\text { swelling }\end{array}$ & $(\%)$ \\
\hline Observation & 50 & 1 & 0 & $2.0^{*}$ \\
Control & 52 & 1 & 1 & 3.8 \\
\hline${ }^{*} P=0.58>0.05$ (compared with the control group)
\end{tabular}

\section{Therapeutic/clinical effects}

There were 42 patients affected obviously, 7 validity patients and 1 invalidity patient in the treatment group. The total effectiveness was $98.0 \%$. There were 20 patients affected obviously, 22 validity patients and 9 invalidity patients in the control group.

Compared with control group, the clinical effectiveness in the treatment group was significantly better $(p<0.05$, Table 4$)$.

Table 1: General profile of patients

\begin{tabular}{|c|c|c|c|c|c|c|c|}
\hline \multirow{2}{*}{ Group } & \multirow{2}{*}{ Number } & \multicolumn{2}{|c|}{ Sex } & \multirow{2}{*}{ Mean age (years) } & \multirow{2}{*}{ Hypertension } & \multirow{2}{*}{ Diabetes } & \multirow{2}{*}{ Hyperlipidemia } \\
\hline & & Male & Female & & & & \\
\hline Observation & 50 & 27 & 23 & $75.21 \pm 9.28$ & 16 & 11 & 11 \\
\hline Control & 52 & 27 & 25 & $76.48 \pm 3.58$ & 12 & 13 & 10 \\
\hline
\end{tabular}


Table 4: Clinical effects of treatment on patients

\begin{tabular}{lccccc}
\hline Group & Number & Obvious effects & Validity & Invalidity & Total effectiveness (\%) \\
\hline Observation & 50 & $42(84.0 \%)$ & $7(14.0 \%)$ & $1(2.0 \%)$ & $98.0^{*}$ \\
Control & 52 & $20(38.5 \%)$ & $22(42.3 \%)$ & $9(17.3 \%)$ & 80.8 \\
\hline${ }^{*} P=0.021<0.05$ (compared with the control group) & & &
\end{tabular}

Table 5: Changes in the levels of D-dimmer, BNP and NF of patients

\begin{tabular}{lcccccc}
\hline \multirow{2}{*}{ Group } & \multicolumn{2}{c}{ D-dimmer $(\mathbf{u g} / \mathrm{L})$} & \multicolumn{2}{c}{ BNP $(\mathrm{ng} / \mathrm{l})$} & \multicolumn{2}{c}{ NF-kB (ng/L) } \\
\cline { 2 - 7 } & $\begin{array}{c}\text { Before } \\
\text { treatment }\end{array}$ & $\begin{array}{c}\text { After } \\
\text { treatment }\end{array}$ & $\begin{array}{c}\text { Before } \\
\text { treatment }\end{array}$ & $\begin{array}{c}\text { After } \\
\text { treatment }\end{array}$ & $\begin{array}{c}\text { Before } \\
\text { treatment }\end{array}$ & $\begin{array}{c}\text { After } \\
\text { treatment }\end{array}$ \\
\hline Observation & $658.5 \pm 78.2$ & $145.2 \pm 57.8$ & $186.8 \pm 8.5$ & $54.6 \pm 4.4$ & $41.94 \pm 3.8$ & $32.15 \pm 4.1$ \\
Control & $675 . \pm 60.27$ & $261.6 \pm 72.0$ & $182.3 \pm 8.1$ & $76.2 \pm 5.7$ & $42.07 \pm 4.0$ & $37.36 \pm 4.4$ \\
$t$ & 0.081 & 8.181 & 0.075 & 2.271 & 0.156 & 5.629 \\
$P$ & $>0.05$ & $<0.05$ & $>0.05$ & $<0.05$ & $>0.05$ & $<0.05$ \\
\hline
\end{tabular}

Changes in levels of D-dimmer, BNP and NF of patients

Before treatment, there were no significant differences in the levels of D-dimmer, BNP and NF-kB between patients in two groups $(p>0.05)$. However, after treatment, D-dimmer, BNP and NF-kB levels in the observation group were significantly lower than the levels in the control group within the same period ( $p<0.05$; Table 5).

\section{DISCUSSION}

Ischemic cerebral injury after cerebral hemorrhage is a secondary damage disease. If the treatment and intervention are not prompt, it aggravates not only the primary disease, but also significantly promotes incidence of multiple complications, leading to threat to health and life of patients. If the progress of ischemic cerebral injury after cerebral hemorrhage can be controlled promptly, it will be of help towards improving the prognosis of patients. This study tried to find the clinical effects of edaravone injection combined with nimodipine on ischemic cerebral injury after cerebral hemorrhage. It also monitored the influence of the combined treatment on cerebrovascular blood flow and inflammatory factors, so as to provide a basis for clinical treatment of the disease

The auto-regulation function of cerebral blood flow in cerebral hemorrhage patients is damaged. Cerebral blood flow shows linear decrease when cerebral perfusion pressure decreases. Thus, the damaged region is more sensitive to changes in pressure. As a consequence, after cerebral hemorrhage, excessive lowering of pressure may result in

The total clinical effectiveness reached $98 \%$, significantly higher than the effectiveness of acute cerebral ischemia as well as aggravate ischemic cerebral injury $[7,8]$.

In addition, studies have shown that vascular spasm after cerebral hemorrhage can reduce blood supply to ischemic cerebral injury [9]. Local dysfunction in cerebral tissue microcirculation, lactic acid accumulation in tissue, free radical metabolic disorder, and release of large amounts of inflammatory factors aggravate ischemic injury [10]. Therefore, improving cerebral blood-supply, relieving vascular spasm and clearing free radicals are the effective methods for improving ischemic cerebral injury after cerebral hemorrhage.

Edaravone is a brain-protective agent; it can clear free radicals and inhibit lipid peroxidation, thus inhibiting injury to cerebral cells, endothelial cells of vessels and nerve cell oxidation. These can effectively prolong progress of cerebral edema and cerebral infarction and inhibit death of neurons [11]. Nimodipine DHP, a second generation calcium antagonist, can increase cerebral blood flow, but does not influence cerebral metabolic function, neither does it improve vascular spasm in focus region, dilate small vessels of brain or accelerate blood circulation [12].

Thus, it can be seen that edaravone and nimodipine are ideal drugs for treating ischemic cerebral injury after cerebral hemorrhage. This study found that edaravone and nimodipine can significantly improve nervous functional defects scores: NIHSS score in the observation group was lower than that of single nimodipine of the control group. The clinical effects of the two drugs were significant.

single nimodipine. This shows that the combination actually had relatively good effects 
on improving cerebral injury. Edaravone and nimodipine had higher safety and less adverse reactions after medication.

D-dimmer level reflects secondary fibrinolytic hyperactivity. It can be used for diagnosis, for prognosis of state before embolism and embolism formation, and its sensitivity and specificity are relatively high [13]. The D-dimmer levels of patients with ischemic cerebral injury after cerebral hemorrhage in the two groups were higher than normal values, which shows that hypercoagulability in vivo is a pathological reaction to embolism formation. D-dimmer level in the two groups after drug treatment decreased to different levels, but the D-dimmer level of the control group was significantly higher than that in the observation group. Combination of the two drugs was beneficial for improving coagulation of patients.

BNP is a neurological hormone which inhibits the release of $A D H$, promotes urination, sodium removal and vessel dilatation. Plasma BNP of cerebral hemorrhage patients were increased, along with increases in secondary ischemia, anoxia, and cranial pressure. These effects cause compression on the hypothalamus, and increase BNP secretion. In addition, changes in serum BNP are associated with cerebral edema, and are of some significance for judging prognosis of cerebral hemorrhage patients [14]. In this study, BNP level significantly decreased after herb treatment in the observation group, and the effects were significantly better than in the control group. This shows that edaravone and nimodipine can bring about improvement in cerebral ischemia, anoxia and cerebral edema.

Inflammatory reactions of reperfusion can promote secondary cerebral injury. NF-kB is a key factor in the mechanism of inflammationassociated ischemic reperfusion injury. It is believed that vascular endothelial cell injury is one of the mechanisms for initiating ischemic neuron injury [15]. Neutrophils migrate, adhere and infiltrate vascular endothelial cells induced by adhesion molecule, thus inducing inflammatory cascade reaction, finally causing ischemic neuron injury. The current study found that the observation group had lowered NF-kB level. This shows that edaravone and nimodipine exert control over inflammatory reactions, and can function in improving cerebral ischemic reperfusion injury.

\section{Limitations of the study}

In the short term, the combination of parenteral edaravone and nimodipine treatment has high efficiency and safety in mitigating cerebral ischemia as well as cerebral edema and controlling inflammatory reactions. However, the long-term effects of the treatment need to be explored in future.

\section{CONCLUSION}

The results of this study indicate that the combination of edaravone and nimodipine has relatively high efficiency and safety in the treatment of ischemic cerebral injury after cerebral hemorrhage. Thus, the two drugs can be used together for improving nerve function.

\section{DECLARATIONS}

\section{Conflict of Interest}

No conflict of interest associated with this work.

\section{Contribution of Authors}

We declared that this research was done by the authors named in this article and all liabilities pertaining to claims relating to the content of this article will be borne by the authors. Kun Li conceived and designed the study, Zhiling $\mathrm{Ji}$ collected and analyzed the data, Qiuju Li wrote the manuscript. All the authors read and approved this work for publication.

\section{REFERENCES}

1. Huttner H, Kuramatsu J. Aktuelle Therapieziele bei intrazerebralen Blutungen Current treatment concepts in intracerebral hemorrhage. Med Klin Intensivmed Notfmed 2017; 112(8): 695-702.

2. Alerhand $S$, Lay $C$. Spontaneous Intracerebral Hemorrhage. Emerg Med Clin North Am 2017; 35(4): 825-845.

3. Hasegawa S, Hasegawa Y, Miura M. Current Therapeutic Drugs Against Cerebral Vasospasm after Subarachnoid Hemorrhage: A Comprehensive Review of Basic and Clinical Studies. Curr Drug Deliv 2017; 14(6): 843-852.

4. Long $B$, Koyfman A, Runyon MS. Subarachnoid Hemorrhage: Updates in Diagnosis and Management. Emerg Med Clin North Am 2017; 35(4): 803-824.

5. Ahn S-H, Savarraj JP, Pervez M, Jones W, Park J, Jeon S-B, Kwon SU, Chang TR, Lee K, Kim DH. The subarachnoid hemorrhage early brain edema score predicts delayed cerebral ischemia and clinical outcomes. Neurosurg 2017.

6. World Health Organization. Declaration of Helsinki. $\mathrm{Br}$ Med J 1996; 313(7070): 1448-1449.

7. Al-Mufti F, Amuluru K, Smith B, Damodara N, El-Ghanem $M$, Singh IP, Dangayach $N$, Gandhi $C D$. Emerging Markers of Early Brain Injury and Delayed Cerebral 
Ischemia in Aneurysmal Subarachnoid Hemorrhage. World Neurosurg 2017; 107: 148-159.

8. Thorén M, Azevedo E, Dawson J, Egido JA, Falcou A, Ford GA, Holmin S, Mikulik R, Ollikainen J, Wahlgren N. Predictors for Cerebral Edema in Acute Ischemic Stroke Treated With Intravenous Thrombolysis. Stroke 2017; 48(9): 2464-2671.

9. Li Z, Zheng X, Li P, Itoua ESR, Moukassa D, Andely FN. Effects of Acupuncture on mRNA levels of apoptotic factors in perihematomal brain tissue during the acute phase of cerebral hemorrhage. Med Sci Monit 2017; 23 : 1522.

10. Sun $Y$, Shen $Q$, Watts $L T$, Muir ER, Huang S, Yang $G-Y$, Suarez Jl, Duong TQ. Multimodal MRI characterization of experimental subarachnoid hemorrhage. Neurosci 2016; 316: 53-62

11. Lerario MP, Gialdini G, Lapidus DM, Shaw MM, Navi BB, Merkler AE, Lip GY, Healey JS, Kamel H. Risk of ischemic stroke after intracranial hemorrhage in patients with atrial fibrillation. PloS one 2015; 10(12): e0145579.

12. Malinova V, Dolatowski K, Schramm P, Moerer O, Rohde $V$, Mielke D. Early whole-brain CT perfusion for detection of patients at risk for delayed cerebral ischemia after subarachnoid hemorrhage. J Neurosurg 2016; 125(1): 128-136.

13. Rodriguez-Régent $C$, Hafsa $M$, Turc G, Hassen WB, Edjlali $M$, Sermet $A$, Laquay $N$, Trystram $D$, Al-Shareef $F$, Meder J-F. Early quantitative CT perfusion parameters variation for prediction of delayed cerebral ischemia following aneurysmal subarachnoid hemorrhage. Eur Radiol 2016; 26(9): 2956-2963.

14. Terpolilli NA, Feiler $S$, Dienel $A$, Müller $F$, Heumos $N$, Friedrich B, Stover J, Thal S, Schöller K, Plesnila N. Nitric oxide inhalation reduces brain damage, prevents mortality, and improves neurological outcome after subarachnoid hemorrhage by resolving early pial microvasospasms. J Cereb Blood Flow Metab 2016; 36(12): 2096-2107.

15. Jiang S, Fei A, Peng Y, Zhang J, Lu Y-r, Wang H-r, Chen $M$, Pan S. Predictors of outcome and hemorrhage in patients undergoing endovascular therapy with solitaire stent for acute ischemic stroke. PLoS One 2015; 10(12): e0144452. 